\title{
When are Static Superhedging Strategies Optimal?
}

\author{
Nicole Branger $\ddagger$ \\ Angelika Esser ${ }^{\ddagger}$ \\ Christian Schlag $\ddagger$
}

Current Version: January 16, 2004

\footnotetext{
${ }^{\ddagger}$ Faculty of Economics and Business Administration, Goethe University, Mertonstr. 17, Uni-Postfach 77, D-60054 Frankfurt am Main, Germany, e-mail: branger@wiwi.uni-frankfurt.de, esser@wiwi.unifrankfurt.de, schlag@wiwi.uni-frankfurt.de.
} 


\title{
When are Static Superhedging Strategies Optimal?
}

\author{
January 16, 2004
}

\begin{abstract}
This paper deals with the superhedging of derivatives on incomplete markets, i.e. with portfolio strategies which generate payoffs at least as high as that of a given contingent claim. The simplest solution to this problem is in many cases a static superhedge, i.e. a buy-and-hold strategy generating an affine-linear payoff.

We study whether a superhedge can be achieved with less initial capital if we also allow for dynamic trading strategies. The answer to this question depends on the kind of the non-traded risk factors. Our main findings for a stochastic volatility model with unbounded volatility show that there is always an optimal static superhedge. Additionally, there may be infinitely many optimal dynamic superhedges which require the same initial capital. In a model with stochastic jumps, it is always either a dynamic or a static strategy which is optimal, but never both. In a model with a stochastic short rate the properties of the interest rate process are also relevant. When there are no bounds for the interest rate optimal superhedges (if they exist) are always static, since the strategy will never contain an investment in the money market account. On the other hand, when interest rates are either bounded or non-negative either a static or a dynamic strategy is optimal, depending on the respective contingent claim.

Our results have important implications for the design of superhedges as they show under which conditions we can restrict the analysis to static strategies. There is no such thing as the incomplete market when it comes to superhedging. Although in continuous-time models the class of possible trading strategies contains much more elements than just static strategies, there is a number of cases where buy-and-hold is as good as or even superior to dynamic strategies.
\end{abstract}

Keywords: Incomplete markets, superhedging, stochastic volatility, stochastic jumps, stochastic interest rates

JEL: G13 


\section{Introduction and Motivation}

In this paper we consider the superhedging of path-independent European contingent claims. A superhedge is a portfolio strategy which generates a payoff at least as high as that of the claim. The key question is whether the cheapest superhedge, i.e. the cheapest strategy generating such a payoff, is static or dynamic. A static superhedge generates an affine-linear dominating payoff. In most cases it is easy to find and easy to implement. Once we have found such a static superhedge we have the question arises whether there is an even cheaper superhedge if we allow for dynamic trading strategies. The answer to this question is also relevant for the determination of price bounds for contingent claims. If a static superhedge is optimal, then the trivial model-independent price bounds cannot be improved upon.

A number of papers are related to the topic of this study. The paper closest to ours is certainly Cvitanic, Pham, and Touzi (1999) who analyze superhedging in stochastic volatility (SV) models. They derive viscosity solutions to partial differential equations, which then give the value process of the cheapest superhedge for the given claim. The general topic of attainability of certain types of claims in incomplete market models is treated in Branger, Esser, and Schlag (2003) and indirectly in Romano and Touzi (1997). In the latter paper it is shown that in an SV model where volatility follows a one-dimensional diffusion the market can be completed by any convex traded payoff. An important application of the results derived in these papers concerns the bounds on prices of contingent claims which are not attainable. In his seminal paper Merton (1973) derives model-independent (and thus static) no-arbitrage bounds for the prices of European and American options. Frey and Sin (1999) demonstrate that these trivial bounds for call prices are the tightest ones for a large class of SV models, while Eberlein and Jacod (1997) obtain a similar result when the stock price is driven by a Lévy process.

In this paper we investigate the superhedging of European path-independent claims in a Markovian setup. Our proofs concerning the optimality of static or dynamic strategies rely on the explicit construction of the optimal superhedge. We derive restrictions for the 
optimality of a strategy, that is we derive conditions a given strategy has to satisfy to be a sound candidate for a superhedge. As an example consider the case of a stochastic interest rate (SI) model with a non-negative short rate, like the short rate model of Cox, Ingersoll, and Ross (1985). If interest rates can go to infinity, we will not take a short position in the money market account since such a position could basically create an infinite liability.

The restrictions for a sound superhedging strategy in the respective model translate into some optimal dominating payoff, which generally characterizes the cheapest superhedge for a given claim. The replicating strategy for this dominating payoff in some worst-case model then produces a superhedge. The intuition for this is that we construct a hedging strategy for the claim under the worst-case scenario so that in any other scenario the strategy never requires an additional infusion of cash, but rather generates a cash outflow in some states of the world.

Our paper contributes to the existing literature in several ways. To the best of our knowledge there has been no paper which analyzes different sources of incompleteness, i.e. we study markets where incompleteness is caused by either SV, stochastic jumps (SJ), or SI (and, ultimately, also by all these factors simultaneously). The key finding here is that it is very important for the optimality of static and dynamic strategies which risk factors are non-traded. The structure of the superhedge, in particular the issue whether the optimal superhedge is static or dynamic, depends on both the model and on the claim under consideration. This will also be demonstrated by means of examples.

While our basic results for the SV economy are the same as those derived by Cvitanic, Pham, and Touzi (1999), we take a different approach to the proofs with a direct focus on stochastic and partial differential equations and their economic interpretation. We show that with unbounded volatility there is always an optimal static superhedge, so dynamic strategies do not offer an improvement over buy-and-hold. Depending on the claim the superhedge may not be unique, in that in addition to the static superhedge there are also dynamic superhedging strategies requiring the same initial investment. This is due to the fact that the worst-case model in the SV setup is a degenerated one with no stochastic movement. These findings extend the main result in Cvitanic, Pham, and Touzi (1999). 
For the sake of completeness, although we do not discuss this scenario in our paper, it should be mentioned that when volatility is bounded as in Avellaneda, Levy, and Parás (1995) the value of the superhedge is given by the solution to a Black-Scholes-Barenblatt equation, and a static superhedge is in general not optimal.

In an economy characterized by stochastic jumps either a static or a dynamic superhedge is optimal for any given claim, but there will never be the case described above for SV models that both types of superhedges can be optimal at the same time. Incompleteness can also be introduced into a pricing model via a stochastic short rate. Although there is by now a wide class of models with a complete market segment for interest sensitive assets, like the Heath-Jarrow-Morton family of models, approaches with only a stochastic short rate are still popular in equity option pricing. For example, the very general option pricing model developed by Bakshi, Cao, and Chen (1997) contains interest rate risk as a factor, and the stochastic behavior of the short rate is modeled by a square root process suggested in Cox, Ingersoll, and Ross (1985). For the subject of superhedging the type of short rate process assumed in the given model is of crucial importance. When interest rates are unbounded, as it is the case in a Gaussian short model like the one proposed by Vasicek (1977), only static superhedges where we just hold the stock are optimal. The intuition for this result is that the superhedging strategy will contain neither a long nor a short position in the money market account, since the short position can drop in value to minus infinity, and the long position cannot guarantee a positive payoff. With non-negative interest rates, a long position in the money market account will ensure a terminal value at least equal to the initial investment, so that now a long position can be used to superhedge a constant. However, it will still be the case that either a static or a dynamic superhedge is optimal, but never both. Finally, with bounded interest rates, in general only dynamic superhedges will be optimal.

To highlight the fact that the optimality of static or dynamic strategies depends on both the model setup and the type of claim, we present some examples of contingent claims for which a superhedge is set up in various incomplete models. We analyze plain vanilla call and put options as convex claims, a 'corporate bond', i.e. the combination of 
the asset and a short put option, as a concave claim, and an option on a power of the stock price with an exponent less than one as a case for a claim which is neither convex nor concave. As indicated above both the type of claim and the type of model are relevant for the optimality of static or dynamic strategies.

Due to the popularity of 'bigger' models with combinations of SV, SJ, and SI like in Bakshi, Cao, and Chen (1997) we also investigate the optimality of strategies in such a more general framework. It turns out that the conditions for the optimality of a static superhedge in the different models (SV, SJ, SI) have to be met simultaneously, since otherwise only a dynamic strategy can be optimal.

The rest of the paper is organized as follows. In Section 2 we derive the main results. Section 3 gives some examples. Section 4 discusses models with more than one non-traded source of risk. Section 5 contains some concluding remarks.

\section{Superhedging Strategies in Incomplete Markets}

\subsection{General remarks}

In the following we consider a European path-independent claim with a terminal payoff at time $T$ given by $h\left(S_{T}\right)$. Unless otherwise noted we assume that for each investigated claim there is at least one superhedge. The basic model setup consists of Markov processes in incomplete markets, where the incompleteness is caused by either stochastic volatility, stochastic jumps or stochastic interest rates. The only attainable European path-independent claims are affine-linear (or even only linear) in the stock as shown in Branger, Esser, and Schlag (2003), and the hedge criterion for a general claim is to find the cheapest superhedge, i.e. to find the cheapest payoff $X$ with $X \geq h\left(S_{T}\right)$ almost surely.

We define a superhedge to be a trading strategy with terminal payoff $X \geq h\left(S_{T}\right)$ almost surely. We do not assume that the associated trading strategy is self-financing. We only assume that there are no injections of money, i.e. the strategy is allowed to generate 
a cash outflow. Furthermore we do not assume that $X$ is path-independent, i.e. the payoff may depend on the complete history of the state variables from $t=0$ to $t=T$.

The main question in this context is when we can restrict ourselves to static, i.e. to buy-and-hold strategies, and under what scenarios we also have to consider dynamic trading strategies in order to find a cheapest superhedge. In the following a strategy is called dynamic only if there is at least one change in the composition of the portfolio over time, i.e. we would not call a pure buy-and-hold strategy dynamic.

Before going into the details of the different models we briefly want to sketch the intuition behind the proofs of our main theorems and propositions: In each model, we first show how to construct a superhedge, which stands in contrast to papers that maximize the price of a given claim over all equivalent martingale measures. For a claim represented by a certain payoff $h\left(S_{T}\right)$, the cheapest superhedge is characterized by some dominating claim $\widehat{h}$, which is not necessarily attainable (but may be). For this dominating claim $\widehat{h}$, we have to determine the replicating strategy in some worst-case model which is 'artificially' complete. For example, in an SV model the worst-case hedge is based on a volatility of zero, which creates a (degenerate example for a) complete market with no uncertainty at all. This replicating strategy for $\widehat{h}$ in the worst-case model is a superhedge in the true model if $\widehat{h}$ meets certain criteria like, e.g., concavity. As $h$ does not necessarily meet these criteria, we cannot simply choose $\widehat{h}$ to be equal to $h$.

The cheapest superhedge for $h$ is thus found by first determining the optimal dominating payoff $\widehat{h}$ which is the payoff as close as possible to $h$ (so that this optimal payoff has the lowest price in the worst-case model), and, second, by implementing the optimal superhedge for this dominating payoff (which is achieved by using the replicating strategy from the worst-case model). From now on, we always understand a superhedge to be a cheapest superhedge. 


\subsection{Stochastic volatility}

The SV model is given by the stochastic differential equations

$$
\begin{aligned}
& d S_{t}=\mu_{S}\left(t, S_{t}, V_{t}\right) S_{t} d t+V_{t} S_{t} d W_{t}^{S} \\
& d V_{t}=\mu_{V}\left(t, S_{t}, V_{t}\right) d t+\sigma_{V}\left(t, S_{t}, V_{t}\right)\left(\rho d W_{t}^{S}+\sqrt{1-\rho^{2}} d W_{t}^{V}\right)
\end{aligned}
$$

where $-1<\rho<1$ to exclude the degenerate case of deterministic volatility for which the model would be complete, and $\sigma_{V} \not \equiv 0$. Furthermore we assume a deterministic money market account $B$ with dynamics $d B_{t}=r B_{t} d t$. As demonstrated in Branger, Esser, and Schlag (2003) attainable path-independent payoffs in the SV setup have to be affinelinear in the stock price. For a general path-independent payoff $h\left(S_{T}\right)$ we now turn to the problem of finding the superhedge. In the case of bounded volatility it is well known that the price of the superhedge satisfies the Black-Scholes-Barenblatt partial differential equation as shown in Avellaneda, Levy, and Parás (1995). For unbounded volatility the result is given in the next proposition.

Proposition 1 (SV: Superhedge a Concave Dominating Payoff) Let the $S V$ model be given by (1) and (2). Volatility $V$ can take on any value in $\mathbb{R}^{+}$. Then, each superhedge for the payoff $h\left(S_{T}\right)$ is identical to a superhedge for the optimal concave payoff $\widehat{h}$ dominating $h$. This optimal dominating payoff is characterized by having the smallest price among all dominating payoffs in the worst-case model with volatility equal to zero, and the replicating strategy in the worst-case model is a superhedge in the $S V$ model.

To prove the proposition we derive the properties of a superhedge which is in general characterized by a dynamic trading strategy. In particular, we show that the superhedge is equal to the replicating strategy for a certain dominating payoff in the worst case model with zero volatility.

For every point in time $t$ let $F_{t}$ be the smallest amount of money we need for superhedging the payoff $h\left(S_{T}\right)$ from $t$ onwards so that $F$ will generally depend on all possible future paths. Since (1) and (2) describe a Markov model, F can be written as a 
function $f$ depending on $t, S_{t}$, and $V_{t}$ only:

$$
F_{t} \equiv f\left(t, S_{t}, V_{t}\right)
$$

At time $t$ we have to invest $f\left(t, S_{t}, V_{t}\right)$ to run the superhedge. If the current value of the hedge portfolio before reinvesting is higher, funds can be withdrawn.

The number of stocks in the hedge portfolio is given by the hedge ratio $H_{t}$, and the investment into the money market account is equal to $f\left(t, S_{t}, V_{t}\right)-H_{t} S_{t}$. To actually represent a superhedge both $f$ and $H$ have to satisfy certain restrictions. First, the trading gains generated by the hedge portfolio have to be greater than or equal to the change in the required capital $F_{t}$ which implies

$$
r f\left(t, S_{t}, V_{t}\right) d t+H_{t}\left(d S_{t}-r S_{t} d t\right) \geq d f\left(t, S_{t}, V_{t}\right)
$$

Second, the terminal value of the hedge portfolio has to dominate the payoff of the claim:

$$
f\left(T, S_{T}, V_{T}\right) \geq h\left(S_{T}\right)
$$

The left-hand side (LHS) of inequality (3) represents the trading gains when we buy $H_{t}$ stocks and invest $F_{t}-H_{t} S_{t}$ in the money market account, the right-hand side (RHS) gives the change in the required minimal capital. Any non-negative difference between LHS and RHS in (3) can be withdrawn. Applying Itô to the RHS of (3) yields

$$
\begin{aligned}
r f d t+H_{t}\left(d S_{t}-r S_{t} d t\right) \geq & \frac{\partial f}{\partial t} d t+\frac{\partial f}{\partial s} d S_{t}+\frac{\partial f}{\partial v} d V_{t} \\
& +\frac{1}{2} \frac{\partial^{2} f}{\partial s^{2}} V_{t}^{2} S_{t}^{2} d t+\frac{1}{2} \frac{\partial^{2} f}{\partial s^{2}} \sigma_{V}^{2} d t+\frac{\partial^{2} f}{\partial s \partial v} V_{t} S_{t} \sigma_{V} \rho d t
\end{aligned}
$$

which is equivalent to

$$
\begin{aligned}
\left(\frac{\partial f}{\partial s}-H_{t}\right) d S_{t}+\frac{\partial f}{\partial v} d V_{t} & +\left(\frac{\partial f}{\partial t}+\frac{1}{2} \frac{\partial^{2} f}{\partial s^{2}} V_{t}^{2} S_{t}^{2}+\frac{1}{2} \frac{\partial^{2} f}{\partial v^{2}} \sigma_{V}^{2}\right. \\
+ & \left.\frac{\partial^{2} f}{\partial s \partial v} V_{t} S_{t} \sigma_{V} \rho+H_{t} r S_{t}-r f\right) d t \leq 0
\end{aligned}
$$

This last inequality has to hold for all values of $d S_{t}$ and $d V_{t}$, implying

$$
\begin{aligned}
H_{t} & =\frac{\partial f}{\partial s}\left(t, S_{t}, V_{t}\right) \\
\frac{\partial f}{\partial v} & =0
\end{aligned}
$$


and furthermore

$$
\frac{\partial f}{\partial t}+\frac{1}{2} \frac{\partial^{2} f}{\partial s^{2}} V_{t}^{2} S_{t}^{2}+\frac{1}{2} \frac{\partial^{2} f}{\partial v^{2}} \sigma_{V}^{2}+\frac{\partial^{2} f}{\partial s \partial v} V_{t} S_{t} \sigma_{V} \rho+H_{t} r S_{t}-r f \leq 0
$$

Condition (5) shows that the hedge ratio $H_{t}$ has to be equal to the partial derivative of the value function of the price bound with respect to the stock price (similar to a classical delta hedge), so that once we know $f$, we can implement a superhedge. Condition (6) means that the price bound must not depend on the non-traded risk factor volatility (similar to the result for attainability as shown in Branger, Esser, and Schlag (2003)). This implies that the function $f$ can be simplified to

$$
f(t, s, v)=f(t, s)
$$

and all derivatives with respect to $v$ vanish. Together with equations (5) and (6) this implies that inequality (7) simplifies to

$$
\frac{\partial f}{\partial t}+\frac{\partial f}{\partial s} r S_{t}+\frac{1}{2} \frac{\partial^{2} f}{\partial s^{2}} V_{t}^{2} S_{t}^{2} \leq r f
$$

This inequality has to hold for all possible realizations of $V_{t}$, and is therefore equivalent to

$$
\frac{\partial f}{\partial t}+\frac{\partial f}{\partial s} r S_{t}+\frac{1}{2} \sup _{V_{t}}\left\{\frac{\partial^{2} f}{\partial s^{2}} V_{t}^{2}\right\} S_{t}^{2} \leq r f
$$

For $\frac{\partial^{2} f}{\partial s^{2}}\left(t, S_{t}\right)>0$, this inequality can only hold if $V_{t}$ is bounded from above, and the superhedge for this scenario is derived in Avellaneda, Levy, and Parás (1995). For unbounded volatility inequality (9) can thus only be satisfied for $\frac{\partial^{2} f}{\partial s^{2}}\left(t, S_{t}\right) \leq 0$ so that $f$ has to be a concave function of the stock. We further assume that volatility can be arbitrarily close to zero so that inequality (8) reduces to

$$
\frac{\partial f}{\partial t}+\frac{\partial f}{\partial s} r s \leq r f
$$

We denote the difference between the RHS and the LHS by the non-negative function $g(t, s)$ :

$$
\frac{\partial f}{\partial t}+\frac{\partial f}{\partial s} r s+g=r f
$$


Furthermore, we define the function $\widehat{h}$ by $f(T, s)=\widehat{h}(s)$. Then, by applying Feyman-Kac to (10) the function $f$ is found to be given by

$$
f(t, s)=\widetilde{E}\left[\int_{t}^{T} e^{-r(u-t)} g\left(u, S_{u}\right) d u+e^{-r(T-t)} \widehat{h}\left(S_{T}\right) \mid S_{t}=s\right]
$$

where for calculating the expectation $\widetilde{E}$ we use the stochastic process

$$
d S_{t}=r S_{t} d t
$$

which follows directly from (10). As the future stock price is certain in this case and just earns the risk-free rate, the function $f$ simplifies to

$$
f(t, s)=\int_{t}^{T} e^{-r(u-t)} g\left(u, s e^{r(u-t)}\right) d u+e^{-r(T-t)} \widehat{h}\left(s e^{r(T-t)}\right) .
$$

The function $f$ depends on the function $\widehat{h}$ and on the non-negative function $g$. It can be interpreted as the price of a claim where $\widehat{h}$ is the terminal payoff function of this special claim, while the function $g$ can be regarded as its continuous dividend stream. The price is calculated in a model with zero volatility. It is important to note that, since $f$ is concave, its price will be highest in this worst-case model compared to models with non-zero volatility.

The strategy described by $f$ and the hedge ratio $H_{t}=\frac{\partial f}{\partial s}\left(t, S_{t}\right)$ is the replicating strategy for the claim with terminal payoff $\widehat{h}$ in the worst-case model with zero volatility. When volatility is stochastic, the strategy will in general be no longer self-financing. However, the concavity of $f$ ensures that condition (7) is met for all possible realizations of $V$, and so no additional funds will have to be injected at any point in time, whereas sometimes the strategy will generate an outflow.

Each non-negative function $g$ and each terminal payoff $\widehat{h}$ define a candidate function $f$ via equation (12). To represent the desired superhedge the functions have to satisfy some conditions. The original claim has to be dominated, yielding $\widehat{h}(s) \geq h(s), f(t, s)$ has to be concave in $s$, and $f$ also has to be optimal in that $f\left(0, S_{0}\right)$ has to be minimal over all candidate functions. 
From these conditions we can derive that $\widehat{h}(s)$ is a concave function of $s$, since $f(T, s)=\widehat{h}(s)$. Furthermore the optimal $g$ will be identically equal to zero, given that $g$ is non-negative, since any choice other than $g \equiv 0$ would lead to a positive dividend stream and therefore to a higher price for the superhedge. For $g \equiv 0$ the candidate function is given by

$$
f(t, s)=e^{-r(T-t)} \widehat{h}\left(s e^{r(T-t)}\right)
$$

which is indeed concave in $s$. We then choose the dominating concave function $\widehat{h}$ such that the price $f\left(0, S_{0}\right)$ today is minimal. This completes the proof of Proposition 1.

Note that there may be more than one superhedge. If we consider the set of all superhedges, then it is of interest whether this set includes both static and dynamic strategies or only one of the two types. The answer is given in the next two corollaries:

Corollary 1 (SV: Optimality of Static Superhedge) Let the $S V$ model be given by (1) and (2). Volatility $V$ can take on any value in $\mathbb{R}^{+}$. Then, in the set of superhedging strategies, there is at least one static strategy.

To prove the corollary we only have to explicitly construct one static superhedge. From Proposition 1 we know that each superhedge is characterized by the optimal dominating function $\widehat{h}$ for which the initially required capital is minimal. We then set up the static hedge as follows. The initial capital $f\left(0, S_{0}\right)=e^{-r T} \widehat{h}\left(S_{0} e^{r T}\right)$ is used to buy $\frac{\partial f}{\partial s}\left(0, S_{0}\right)=\widehat{h}^{\prime}\left(S_{0} e^{r T}\right)$ stocks, and the rest is invested into the money market account. The payoff at time $T$ of this static strategy is given by

$$
\frac{\partial f}{\partial s}\left(0, S_{0}\right) S_{T}+\left(f\left(0, S_{0}\right)-\frac{\partial f}{\partial s}\left(0, S_{0}\right) S_{0}\right) e^{r T} .
$$

This payoff indeed dominates $h$, since

$$
\begin{aligned}
\frac{\partial f}{\partial s}\left(0, S_{0}\right)\left(S_{T}-S_{0} e^{r T}\right)+f\left(0, S_{0}\right) e^{r T} & =\widehat{h}^{\prime}\left(S_{0} e^{r T}\right)\left(S_{T}-S_{0} e^{r T}\right)+\widehat{h}\left(S_{0} e^{r T}\right) \\
& \geq \widehat{h}\left(S_{T}\right) \\
& \geq h\left(S_{T}\right) .
\end{aligned}
$$


The first inequality follows from the concavity of $\widehat{h}$, the second inequality from the fact $\widehat{h}$ dominates the payoff $h$. The static strategy needs the same initial capital as a superhedge, and the terminal payoff also dominates the claim payoff $h$. Therefore, the static strategy is also a superhedge.

The corollary shows that in an SV model with unbounded volatility we can restrict ourselves to static strategies in order to find a superhedge. For any dynamic trading strategy with a terminal payoff dominating $h$ and with an initially lower price the probability that we would have to inject funds at some point in time would be strictly positive.

Since the proof is very technical, it may be useful to review the intuition behind the analysis presented so far. The main point in finding a superhedge is to determine an optimal dominating payoff $\widehat{h}$. The price of this payoff in the worst case model is the initial capital needed for the superhedge, and the replicating strategy for $\widehat{h}$ in the worst case model is a superhedge for $\widehat{h}$ and therefore also for the smaller payoff $h$ in the original model. In the case of SV with unbounded volatility the only possible worst case is a volatility which is identically equal to zero, and $\widehat{h}$ has to be concave in the stock price. Furthermore, $\widehat{h}$ has to be chosen such that its price in the worst case model is minimal.

In the worst case model, volatility is zero, which implies that the stock price is deterministic. We then 'know' that the terminal stock price will be $S_{0} e^{r T}$, and the price of $\widehat{h}$ in the worst case model at time $t=0$ is $e^{-r T} \widehat{h}\left(S_{0} e^{r T}\right)$.

We now consider an affine-linear payoff which is tangent to $\widehat{h}$ in $S_{T}=S_{0} e^{r T}$. Since $\widehat{h}$ is concave, this affine-linear payoff dominates the payoff $\widehat{h}$ and thus also the smaller payoff $h$. The replicating strategy for this payoff in the worst case model is a static strategy with a terminal payoff dominating $h$. Furthermore, the initial capital for this static strategy is given by $e^{-r T} \widehat{h}\left(S_{0} e^{r T}\right)$, and this coincides with the capital needed for the superhedge implied by $\widehat{h}$. These two properties show that the static strategy is also a superhedge in the original model with non-zero volatility.

So both $\widehat{h}$ and the affine-linear payoff tangent to it at $S_{T}=S_{0} e^{r T}$ define a superhedge. If $\widehat{h}$ is not itself affine-linear, then it is dominated by the affine-linear payoff given 
by the tangent. In this case, it may seem surprising that both payoffs describe a superhedge, since our aim is to find the cheapest claim in the worst case model which should exclude such dominating payoffs. However, in the worst case model, there is only one possible stock price at time $T$, and for this stock price both payoffs coincide, so that in this sense the affine-linear payoff is no longer dominating $\widehat{h}$. The joint optimality of dynamic strategies and static strategies is therefore special to an SV model with unbounded volatility.

Corollary 1 shows that there is a static superhedge in this model. The conditions for the optimality of dynamic superhedges are given in the next corollary.

Corollary 2 (SV: Dynamic Superhedges) If the cheapest dominating payoff $\widehat{h}$ is not affine-linear, then there are infinitely many dynamic superhedging strategies. If $\widehat{h}$ is affinelinear, then there is no dynamic superhedge.

To prove the corollary note that by Proposition 1, the following strategy is a superhedge: At time $t$ the funds needed to run the strategy are given as $f\left(t, S_{t}\right)$, the hedge ratio is $\frac{\partial f}{\partial s}\left(t, S_{t}\right)$. We can now compute the amount of money we can withdraw at time $t$ :

$$
\begin{aligned}
f\left(t, S_{t}\right) r d t+\frac{\partial f}{\partial s}\left(t, S_{t}\right)\left(d S_{t}-r S_{t} d t\right)-d f\left(t, S_{t}\right) \\
=\quad f\left(t, S_{t}\right) r d t+\frac{\partial f}{\partial s}\left(t, S_{t}\right)\left(d S_{t}-r S_{t} d t\right) \\
\quad-\left(\frac{\partial f}{\partial t}\left(t, S_{t}\right) d t+\frac{\partial f}{\partial s}\left(t, S_{t}\right) d S_{t}+\frac{1}{2} \frac{\partial^{2} f}{\partial s^{2}}\left(t, S_{t}\right) V_{t}^{2} S_{t}^{2} d t\right) \\
=\left(f\left(t, S_{t}\right) r-\frac{\partial f}{\partial s}\left(t, S_{t}\right) r S_{t}-\frac{\partial f}{\partial t}\left(t, S_{t}\right)-\frac{1}{2} \frac{\partial^{2} f}{\partial s^{2}}\left(t, S_{t}\right) V_{t}^{2} S_{t}^{2}\right) d t \\
=-\frac{1}{2} \frac{\partial^{2} f}{\partial s^{2}}\left(t, S_{t}\right) V_{t}^{2} S_{t}^{2} d t \\
\geq 0,
\end{aligned}
$$

where we have used the concavity of $f$ and equation (10) with $g \equiv 0$ to conclude that

$$
f\left(t, S_{t}\right) r-\frac{\partial f}{\partial s}\left(t, S_{t}\right) r S_{t}-\frac{\partial f}{\partial t}\left(t, S_{t}\right)=0 .
$$

If $\widehat{h}$ is not affine-linear, $\frac{\partial^{2} f}{\partial s^{2}}$ will not be identically equal to zero, and the probability that we withdraw money is strictly positive. We can invest the free cash flow of this 
strategy into some limited liability asset. The resulting overall trading strategy is again a superhedge. Its terminal payoff is equal to $\widehat{h}\left(S_{T}\right)$ plus some non-negative term . Since there are infinitely many possibilities for such a limited liability investment, there must also be infinitely many dynamic superhedging strategies.

If $\widehat{h}$ is affine-linear, we have to superhedge an affine-linear payoff. In this case, there is even a unique replicating strategy for $\widehat{h}$, and this replicating strategy is obviously static. This precludes the existence of any other superhedge.

In summary for an SV economy with unbounded volatility there is always a static superhedge, given that a superhedge exists at all, that is given that there is a dominating concave payoff. The consideration of dynamic trading strategies does not offer any improvement. If the concave payoff dominating the original claim is affine-linear, there is no dynamic superhedge. Otherwise, there are infinitely many dynamic superhedges.

\subsection{Stochastic jumps}

The SJ model is given by the stochastic differential equation

$$
d S_{t}=\mu_{S}\left(t, S_{t}\right) S_{t} d t+\sigma_{S}\left(t, S_{t}\right) S_{t} d W_{t}+X_{t} S_{t} d N_{t}
$$

The counting process $N$, the Brownian motion $W$ and the jump size $X$, which can take on any value greater than -1 , are assumed to be independent. Furthermore we assume that the money market account is deterministic with dynamics $d B_{t}=r B_{t} d t$. The following proposition shows how to find the superhedge for a given claim:

Proposition 2 (SJ: Superhedge for a Concave Dominating Payoff) Let the SJ model be given by (13). In this model a superhedge for the payoff $h\left(S_{T}\right)$ is identical to a superhedge for an optimal concave payoff $\widehat{h}$ dominating $h$. The optimal dominating payoff is characterized by the smallest price in the worst case model with no jumps, and the replicating strategy in the worst case model is a superhedge in the SJ model. 
For the proof of this proposition let again $F_{t}$ be the smallest amount of money we need to superhedge the payoff $h$ from time $t$ onwards, and let $H_{t}$ be the associated hedge ratio. Since (13) describes a Markov model, $F_{t}$ can be written as a function of $t$ and $S_{t}$ :

$$
F_{t}=f\left(t, S_{t}\right)
$$

As in the proof of Proposition 1, the conditions for $f$ and $H$ to describe a superhedge are that the trading gains of the hedge portfolio are greater than or equal to the change in the lower bound $f$ and that the terminal value of the strategy dominates the claim. These conditions can be written as

$$
r f\left(t, S_{t}\right) d t+H_{t}\left(d S_{t}-r S_{t} d t\right) \geq d f\left(t, S_{t}\right)
$$

and

$$
f\left(T, S_{T}\right) \geq h\left(S_{T}\right)
$$

Applying Itô to the RHS of (14) - the formula can for example be found in Duffie (2001, p. 348) - and rearranging terms yields

$$
\begin{aligned}
\left(\frac{\partial f}{\partial s}\left(t, S_{t-}\right)-H_{t}\right) d S_{t} & +\left\{f\left(t, S_{t-}+S_{t-} X_{t}\right)-f\left(t, S_{t-}\right)-\frac{\partial f}{\partial s}\left(t, S_{t-}\right) X_{t} S_{t-}\right\} d N_{t} \\
+ & \left(\frac{\partial f}{\partial t}\left(t, S_{t-}\right)+\frac{1}{2} \frac{\partial^{2} f}{\partial s^{2}}\left(t, S_{t-}\right) \sigma_{S}\left(t, S_{t-}\right)^{2} S_{t-}^{2}+H_{t} r S_{t-}-r f\left(t, S_{t-}\right)\right) d t \leq 0 .
\end{aligned}
$$

This inequality has to hold for all values of $d S_{t}, X_{t}$, and $d N_{t}$ implying

$$
\begin{aligned}
H_{t} & =\frac{\partial f}{\partial s}\left(t, S_{t-}\right), \\
f\left(t, S_{t-}+S_{t-} X_{t}\right)-f\left(t, S_{t-}\right)-\frac{\partial f}{\partial s}\left(t, S_{t-}\right) X_{t} S_{t-} & \leq 0 .
\end{aligned}
$$

To obtain inequality (16), note that $d N_{t}$ is either 0 or 1 . Furthermore, $f$ has to satisfy

$$
\frac{\partial f}{\partial t}\left(t, S_{t-}\right)+\frac{\partial f}{\partial s}\left(t, S_{t-}\right) r S_{t-}+\frac{1}{2} \frac{\partial^{2} f}{\partial s^{2}}\left(t, S_{t-}\right) \sigma_{S}\left(t, S_{t-}\right)^{2} S_{t-}^{2} \leq r f\left(t, S_{t-}\right),
$$

where we have already used the result from equation (15).

From (16) we can conclude that $f(t, s)$ is a concave function of $s$. As above in the case of SV we denote the difference between the RHS and the LHS of (17) by the non-negative 
function $g(t, s)$ :

$$
\frac{\partial f}{\partial t}(t, s)+\frac{\partial f}{\partial s}(t, s) r s+\frac{1}{2} \frac{\partial^{2} f}{\partial s^{2}}(t, s) \sigma_{S}(t, s)^{2} s^{2}+g(t, s)=r f(t, s) .
$$

Furthermore, we define $\widehat{h}$ by $\widehat{h}(s)=f(T, s)$. Then, by Feyman-Kac, the function $f$ is given by

$$
f(t, s)=\widetilde{E}\left[\int_{t}^{T} e^{-r(u-t)} g\left(u, S_{u}\right) d u+e^{-r(T-t)} \widehat{h}\left(S_{T}\right) \mid S_{t}=s\right],
$$

where the dynamics of $S$ are given by

$$
d S_{t}=r S_{t} d t+\sigma_{S}\left(t, S_{t}\right) S_{t} d \widetilde{W}_{t}
$$

i.e. without the jump component. The function $f$ depends on the function $\widehat{h}$ and on the non-negative function $g$. We can interpret $f$ as the price of a claim with terminal payoff $\widehat{h}$ and dividend stream $g$ in a model with no jumps given by (19), representing again the worst-case model. Note that this worst-case model is complete, with volatility being a deterministic function of $t$ and $S_{t}$.

Since $f$ is concave, the price of the claim is maximal under the assumption of no jumps. Formally, this is derived from the fact that the price of a concave claim is a decreasing function of the jump intensity. To see this intuitively, note that for pricing purposes the presence of jumps can be regarded as an increase in volatility, and the value of a concave claim is maximal at the lower volatility bound. The trading strategy given by $f$ and $H_{t}$ is the replicating strategy for the payoff $\widehat{h}$ in the worst-case model. In the true model with jumps, this strategy is of course no longer replicating. However, the concavity of $f$ ensures that there are only withdrawals, but no injections of money (which is just condition (14)).

Each non-negative function $g$ and each terminal condition $\widehat{h}$ define by (18) a candidate function $f$. As in the SV case the functions that describe the superhedge are characterized by the fact that $\widehat{h}\left(S_{T}\right) \geq h\left(S_{T}\right)$, by the concavity of $f(t, s)$ with respect to $s$, and by the optimality of $f\left(0, S_{0}\right)$, which has to be minimal over all possible choices of functions. 
From these conditions we get that, since $f$ has to be concave, $f(T, s)$ and therefore also $\widehat{h}(s)$ are concave functions of $s$. Given a concave and dominating function $\widehat{h}$, the optimal $g$ is equal to zero. To see this, note that for $g$ identically equal to zero the candidate function is given by

$$
f(t, s)=\widetilde{E}\left[e^{-r(T-t)} \widehat{h}\left(S_{T}\right) \mid S_{t}=s\right]
$$

which is indeed concave in $s$. This follows from what Bergman, Grundy, and Wiener (1996) call inherited concavity, i.e. the fact that the pricing function inherits concavity from the terminal payoff. For any choice of the non-negative dividend stream $g$ other than $g \equiv 0$, the resulting candidate function is greater so that it cannot be optimal. Finally, we have to choose the dominating function $\widehat{h}$ such that the price $f\left(0, S_{0}\right)$ today is minimal where the price is calculated in the worst case model with no jumps.

Now, the question arises whether the set of superhedges contains both static and dynamic strategies or just one of the two types. The following corollary provides the answer.

Corollary 3 (SJ: Either Static or Dynamic Superhedge) Let the SJ model be given by (13). If the cheapest dominating claim $\widehat{h}$ is affine-linear, then the superhedge is static, and there is no dynamic superhedge. If $\widehat{h}$ is not affine linear, then there are infinitely many dynamic superhedging strategies, but there is no static superhedge.

The proof builds on Proposition 2, which shows that we obtain a superhedge if we use the unique replicating strategy in the worst case model. We now have to check whether there are more superhedges than these fundamental ones.

The replicating strategy in the worst-case model is given by the required capital at time $t, f\left(t, S_{t-}\right)$, and the hedge ratio $\frac{\partial f}{\partial s}\left(t, S_{t-}\right)$. If a jump occurs over the next interval, then we may be able to withdraw money immediately afterwards, since

$$
\begin{aligned}
& f\left(t, S_{t-}\right) r d t+\frac{\partial f}{\partial s}\left(t, S_{t-}\right)\left(d S_{t}-r S_{t-} d t\right)-d F_{t} \\
& \quad=-\left\{f\left(t, S_{t-}+S_{t-} X_{t}\right)-f\left(t, S_{t-}\right)-\frac{\partial f}{\partial s}\left(t, S_{t-}\right) X_{t} S_{t-}\right\} d N_{t}
\end{aligned}
$$


and the term in brackets is less than or equal to zero due to the concavity of $f$. Furthermore, $d N_{t}$ is non-negative (it is either 0 or 1 ), so that indeed no additional funds are required along any path from $t=0$ to $t=T$.

If $\widehat{h}$ is not affine-linear, the term in brackets is not identically equal to zero, and the probability at time $t$ that we can withdraw money an instant after time $t$ is strictly positive. In this case, we can invest the free cash flow into some limited liability strategy, which again results in a superhedge. Since there are infinitely many such limited liability strategies, there are also infinitely many dynamic superhedges.

If the dominating payoff $\widehat{h}$ is affine-linear, the term in brackets is identically equal to zero. There are no intermediate withdrawals of money, and therefore, there is only the static superhedge, but no dynamic superhedge.

\subsection{Stochastic interest rates}

The SI model is represented by the stochastic differential equations

$$
\begin{aligned}
d S_{t} & =\mu_{S}\left(t, S_{t}, R_{t}\right) S_{t} d t+\sigma_{S}\left(t, S_{t}\right) S_{t} d W_{t}^{S} \\
d R_{t} & =\mu_{R}\left(t, S_{t}, R_{t}\right) d t+\sigma_{R}\left(t, R_{t}\right)\left(\rho d W_{t}^{S}+\sqrt{1-\rho^{2}} d W_{t}^{R}\right)
\end{aligned}
$$

where $-1<\rho<1$ to ensure that the market is incomplete. Although there are by now a number of models with a complete market segment for interest sensitive assets, like the Heath-Jarrow-Morton family, short rate models are still popular in equity option pricing, with a prominent example given by the general model derived in Bakshi, Cao, and Chen (1997). So models of the type represented by equations (20) and (21) are still worth investigating. As mentioned in Branger, Esser, and Schlag (2003) attainable pathindependent payoffs in this model are linear, not affine-linear, in the stock price, since the constant cannot be replicated.

First we consider the case of an unbounded short rate (SIUSR)

Proposition 3 (SIUSR: Superhedge) Let the SI model be given by (20) and (21). 
Assume that the short rate $R_{t}$ can take on any value in $\mathbb{R}$. Then the superhedge for the claim $h\left(S_{T}\right)$ is identical to the superhedge for the linear claim $\widehat{h} \geq h$ with minimal price.

Like in the proof of Proposition 1, let $F_{t}$ be the smallest amount we need for superhedging the payoff $h$ from time $t$ onwards, and let $H_{t}$ be the hedge ratio. (20) and (21) describe a Markov model, so $F_{t}$ is a function of $t, S_{t}$, and $R_{t}$ only:

$$
F_{t}=f\left(t, S_{t}, R_{t}\right)
$$

The condition that the trading gains of the hedge portfolio are greater than or equal to the change in the lower bound $F_{t}$ can be written as

$$
R_{t} f\left(t, S_{t}, R_{t}\right) d t+H_{t}\left(d S_{t}-R_{t} S_{t} d t\right) \geq d f\left(t, S_{t}, R_{t}\right)
$$

This inequality implies, as usual, that no additional funds are needed, but in some scenarios money can be withdrawn from the hedge portfolio. Furthermore the terminal value has to be greater than or equal to the payoff $h$, i.e.

$$
f\left(T, S_{T}, R_{T}\right) \geq h\left(S_{T}\right)
$$

Applying Itô to the RHS of (22) and rearranging gives

$$
\begin{aligned}
\left(\frac{\partial f}{\partial s}-H_{t}\right) & d S_{t}+\frac{\partial f}{\partial r} d R_{t}+\left(\frac{\partial f}{\partial t}+\frac{1}{2} \frac{\partial^{2} f}{\partial s^{2}} \sigma_{S}^{2} S_{t}^{2}\right. \\
& \left.+\frac{1}{2} \frac{\partial^{2} f}{\partial r^{2}} \sigma_{R}^{2}+\frac{\partial^{2} f}{\partial s \partial r} \sigma_{S} S_{t} \sigma_{R} \rho+H_{t} R_{t} S_{t}-R_{t} f\right) d t \leq 0 .
\end{aligned}
$$

The inequality has to hold for all values of $d S_{t}$ and $d R_{t}$ so that

$$
\begin{aligned}
H_{t} & =\frac{\partial f}{\partial s}\left(t, S_{t}, R_{t}\right), \\
\frac{\partial f}{\partial r}\left(t, S_{t}, R_{t}\right) & =0
\end{aligned}
$$

implying that $f$ does not depend on the short rate. We can thus write $f(t, s, r)=f(t, s)$. Furthermore, with (23) and (24) we obtain

$$
\frac{\partial f}{\partial t}+\frac{\partial f}{\partial s} R_{t} S_{t}+\frac{1}{2} \frac{\partial^{2} f}{\partial s^{2}} \sigma_{S}^{2} S_{t}^{2} \leq R_{t} f
$$


which is equivalent to

$$
\frac{\partial f}{\partial t}+\left(\frac{\partial f}{\partial s} S_{t}-f\right) R_{t}+\frac{1}{2} \frac{\partial^{2} f}{\partial s^{2}} \sigma_{S}^{2} S_{t}^{2} \leq 0
$$

In the case of unbounded interest rates, e.g. in a Gaussian model like Vasicek (1977), inequality (25) can only hold if

$$
f(t, s)-\frac{\partial f}{\partial s}(t, s) s \equiv 0
$$

so $f$ is linear in $s$, and $f(t, s)=b(t) s$, where $b$ is at most a deterministic function of time. Then, the last term on the LHS of equation (25) vanishes and the inequality can indeed be solved by a linear function.

The superhedge amounts to holding $b(t)$ stocks at time $t$. It can easily be seen that $b(t)$ has to be constant, since otherwise we would need additional funds at some point in time to buy more units of the stock (and these funds are not available) or we would have already held too much of the stock at time $t$ (so that the strategy would not have been the cheapest possible hedge). This shows that we can set $b(t) \equiv \beta$, where $\beta$ is chosen such that $\beta s \geq h(s)$ and $\beta S_{0}$ is as small as possible.

Note that restriction (26) basically says that there must be no investment in the money market account. This makes sense, since interest rates are not bounded from below. Each positive investment in the money market account could thus drop to zero. However, to guarantee a payoff of zero, it is cheaper to do nothing than to invest in the money market account. Analogously, since interest rates are not bounded from above, each negative investment in the money market account can go to minus infinity. So with the stock only one of the two traded instruments will be included in our hedge portfolio, which automatically implies that the strategy has to be static. Thus, we have already proved the following corollary:

Corollary 4 (SI with Unbounded Short Rate: Only Static Superhedge) Let the SI model be given by (20) and (21). The short rate $R_{t}$ can take on any value in $\mathbb{R}$. Then, any superhedge for a payoff $h\left(S_{T}\right)$ is a static strategy. 
If interest rates are bounded below by zero in an SI model with non-negative short rates (SINSR), the results change. In this case, also dynamic strategies can be optimal. We start with the characterization of the superhedge:

Proposition 4 (SINSR: Superhedge) Let the SI model be given by (20) and (21). Assume that the short rate $R_{t}$ can take on any value in $\mathbb{R}^{+}$. Then, each superhedge for the payoff $h\left(S_{T}\right)$ is equal to a superhedging strategy for the optimal dominating payoff $\widehat{h}$ for which

$$
\widehat{h}(s)-\frac{\partial \widehat{h}(s)}{\partial s} s \geq 0
$$

The optimal dominating payoff is characterized by the smallest price in the worst case model with zero interest rates, and the replicating strategy in the worst case model is a superhedge in the SI model. Finally, for $\widehat{h}(0)=0$ the function $\widehat{h}$ is concave.

The first part of the proof is identical to the proof of Proposition 3. Again, inequality (25) has to hold, which in a scenario of non-negative unbounded rates implies

$$
f(t, s)-\frac{\partial f}{\partial s}(t, s) s \geq 0
$$

Using this result we can rewrite inequality (25) as

$$
\frac{\partial f}{\partial t}(t, s)+\frac{1}{2} \frac{\partial^{2} f}{\partial s^{2}}(t, s) \sigma_{S}^{2} s^{2} \leq 0
$$

Again, we introduce a non-negative function $g(t, s)$ to enforce equality:

$$
\frac{\partial f}{\partial t}(t, s)+\frac{1}{2} \frac{\partial^{2} f}{\partial s^{2}}(t, s) \sigma_{S}^{2} s^{2}+g(t, s)=0
$$

where we can interpret $\widehat{h}\left(S_{T}\right)=f\left(T, S_{T}\right)$ as a terminal payoff and $g$ again as a dividend stream. The function $f$ can be seen as the price of the claim in a model with zero interest rates, which is the (complete) worst case model in this setting.

Note that inequality (28) says that the investment in the money market account must be non-negative. Any negative investment in the money market account can drop in value to minus infinity if interest rates increase without bounds, which is the same 
argument as the one used above in the case of unbounded rates. A positive investment in the money market account earns an interest rate of zero in the worst case. So a positive investment in the money market account can at least guarantee a constant payoff at time $T$.

The same line of arguments as in the proof of Proposition 1 now shows that the function $g$ is identically equal to zero, which reduces the partial differential equation (29) to

$$
\frac{\partial f}{\partial t}(t, s)+\frac{1}{2} \frac{\partial^{2} f}{\partial s^{2}}(t, s) \sigma_{S}^{2} s^{2}=0
$$

For the function $\widehat{h}$, the inequality

$$
\widehat{h}(s)-\frac{\partial \widehat{h}(s)}{\partial s} s \leq 0
$$

has to hold. Then, for the function $f$, inequality (28) and therefore also inequality (25) hold. This ensures that the replicating strategy for $\widehat{h}$ in the worst case model never needs additional funds in the original SI model with non-negative interest rates.

We now show that either a static or a dynamic superhedge will be optimal, but never both.

Corollary 5 (SINSR: Static or Dynamic Superhedge) Let the SI model be given by (20) and (21). Assume that the short rate $R_{t}$ can take on any value in $\mathbb{R}^{+}$. If for a payoff $h$ the cheapest dominating payoff $\widehat{h}$ is an affine-linear function of the stock price, then there is only a static superhedges. Otherwise, there are no static, but infinitely many dynamic superhedges.

By Proposition 4, we get a superhedge if we use the unique replicating strategy for $\widehat{h}$ in the worst case model with zero interest rates. It is static if $\widehat{h}$ is affine-linear, and it is dynamic otherwise. It remains to be checked whether there are other superhedges.

The replicating strategy in the worst-case model is characterized by the necessary capital at time $t, f\left(t, S_{t}\right)$, and the hedge ratio $\frac{\partial f}{\partial s}\left(t, S_{t}\right)$. The amount of money we can 
withdraw from our hedge portfolio an instant afte time $t$ is given by

$$
f\left(t, S_{t}\right) R_{t} d t+\frac{\partial f}{\partial s}\left(t, S_{t}\right)\left(d S_{t}-R_{t} S_{t} d t\right)-d f\left(t, S_{t}\right)=R_{t}\left(f\left(t, S_{t}\right)-\frac{\partial f}{\partial s}\left(t, S_{t}\right) S_{t}\right) d t
$$

This is just the interest earned on the position in the money market account. As this position has to be non-negative, the RHS is also non-negative.

When $\widehat{h}$ is not affine-linear, the term is brackets is not identically equal to zero, so that there will be some scenarios under which we can take funds out of the hedge and invest it into some other strategy with limited liability, implying that there are infinitely many dynamic superhedges. If on the other hand the dominating payoff $\widehat{h}$ is affine-linear, the term in brackets is identically equal to zero. This means that we cannot withdraw funds from the hedge, and the only superhedge is static.

The case of a bounded short rate is analogous to the scenario studied Avellaneda, Levy, and Parás (1995) for a bounded volatility. In the latter setup, the claim is hedged at the upper volatility bound for a positive gamma and at the lower volatility bound for a negative gamma. Here, we switch between the upper and the lower bound on interest rates. The worst case is given by the upper bound when there is a short position in the money market account, and it is given by the lower bound for a long position in the money market account.

\section{Examples}

The analysis in Section 2 is rather technical, so that some examples may help to clarify the structural differences between the respective sources of incompleteness when it comes to superhedging. The key finding of the previous section certainly is that the optimality of static or dynamic superhedges is largely determined by which risk factors are non-traded. This means that in different incomplete models the superhedges for the same claim can be fundamentally different.

We look at superhedges for a number of different derivative contracts in the various types of incomplete markets (SV, SJ, SI) discussed before: Standard call and put options 
represent claims which are convex in the stock price, whereas a position consisting of the underlying plus a short put is concave. In a firm value model for defaultable securities this claim would represent the payoff of a corporate bond. Finally, we also consider a claim which is neither convex nor concave, namely a call option on a power of the stock price with an exponent less than one. The results of this section are summarized in Table 1.

\subsection{Call}

It turns out that in all of the incomplete models analyzed here there is no dynamic superhedge for the call payoff $h\left(S_{T}\right)=\left(S_{T}-X\right)^{+}$, so that we are left with the trivial static superhedge, which consists of holding the stock. In the case of an SV model with unbounded volatility the optimal dominating concave payoff for the convex call is given

by the affine-linear function $\widehat{h}(s)=s$. This gives a static superhedge, and from Corollary 2 we can conclude that there is no dynamic superhedge.

In the SJ model the dominating payoff has to be concave according to Proposition 2, which yields the stock as the dominating claim, implying a static, but no dynamic superhedge, as can be derived from Corollary 3.

In an SI model with unbounded interest rates the dominating payoff has to be the stock, since the optimal superhedge will not contain an investment in the money market account. When interest rates are non-negative the dominating payoff will again be the stock, since the replicating strategy in the worst-case model with a zero interest rate must not involve a short position in the money market account according to Proposition 4. This condition would not be met by the call itself, since the call is a levered investment in the stock, and a short position in the money market account is needed. However, the condition is met by a hedge which consists of the stock only. The fact that there is no dynamic superhedge follows from Corollary 5 . 


\subsection{Put}

Although calls and puts are both convex claims the fact that there is a finite upper bound for the payoff of a put, $h\left(S_{T}\right)=\left(X-S_{T} 2\right)^{+}$, creates substantial differences between the superhedges for the two types of options in the various incomplete models. There is just a static superhedge for the put in the SV and SJ case. In the SI model with non-negative interest rates all superhedges are dynamic, and in the case of unbounded interest rates there is no superhedge at all.

In the SV model with unbounded volatility the dominating concave payoff is the constant payoff equal to the strike price, i.e. $\widehat{h}(s)=X$. Since the put is convex, the cheapest dominating concave payoff is affine-linear with a static, but no dynamic superhedge according to Corollary 2.

In the SJ model the analysis is structurally equal to the SV case. The dominating payoff is again the constant $X$ with the same implications for the superhedge as those presented for the SV case.

In the SI model with unbounded interest rates, we obtain the extreme result that there is no superhedge for a standard put. The intuition here is that the maximum payoff of the put is equal to the strike price $X$ for $S_{T}=0$. In the given model there is no way to guarantee this payoff, because the stock obviously pays zero in this case, and the money market account cannot guarantee a positive payoff either, since interest rates can go to minus infinity. With non-negative interest rates the dominating payoff is the put itself, since it satisfies the condition stated in inequality (27) in Proposition 4. The structure of the dominating payoff implies a dynamic superhedge from which for $R>0$ money can be withdrawn and reinvested into a limited liability strategy. This generates infinitely many dynamic superhedges, but no static superhedge as stated in Corollary 5. Note the structural difference to the call for which there is a static, but no dynamic superhedge. 


\subsection{Corporate Bond}

The terminal payoff structure of the corporate bond is given by $h(s)=X-(X-s)^{+}=$ $\min \{s, X\}$, i.e. $h$ is concave in $s$. Again, we obtain different superhedges in the different models. When interest rates are stochastic and unbounded there is only a static superhedge, whereas in the case of non-negative interest rates or with jumps, only dynamic superhedges exist. Finally, in the SV economy both dynamic and static superhedges exist.

In the SV case we have to find a concave dominating claim, which is in this case equal to the claim itself. Dynamic superhedges for this claim exist according to Corollary 2 , since in the worst case model with zero volatility money can be withdrawn whenever the true volatility is greater than zero. The existence of a static superhedge follows from Corollary 1. There is no more uncertainty in the worst-case model, so the strategy has to yield a payoff $h\left(S_{0} e^{r T}\right)$ for the deterministic future stock price $S_{0} e^{r T}$, and the payoff of the strategy has to dominate $h$ in the original model. For $S_{0} e^{r T}<X$ the static superhedge is unique and consists of one unit of the stock, for $S_{0} e^{r T}>X$ it is also unique with an investment equal to $X e^{-r T}$ into the money market account. For the special case $S_{0} e^{r T}=X$ it is not unique, since we can buy $\alpha$ shares of stock with $\alpha \in[0,1]$, and invest the remaining funds into the money market account.

In the SJ model the dominating payoff is also the corporate bond itself, i.e. $\widehat{h}(s)=$ $\min \{s, X\}$. The superhedge given by the replicating strategy in the worst-case model is dynamic, and we can withdraw funds whenever there is a jump so that there are infinitely many dynamic superhedges. Corollary 3 shows that there is no static superhedge, since $\widehat{h}$ is not affine-linear.

With unbounded stochastic interest rates the dominating payoff is the stock, since the superhedge must not contain a position in the money market account. By the same token the superhedge must be static, implying on the other hand that there is no dynamic superhedge.

When interest rates are non-negative the dominating payoff is the corporate bond 
itself, since the only restriction on the replicating strategy in the worst-case model with a zero interest rate is that it must not involve a short position in the money market account as shown in Proposition 4. This already holds for the corporate bond itself. The superhedge is therefore dynamic, and in addition, Corollary 5 tells us that there is no static superhedge.

\subsection{Power Call}

A power call has a payoff equal to either $\left(S_{T}^{\gamma}-X\right)^{+}$or $\left[\left(S_{T}-X\right)^{+}\right]^{\gamma}$ with $\gamma>0$. When $\gamma>1$ there is no superhedge at all, since there is no dominating payoff (neither concave nor linear) in either of the models. So the interesting case is $\gamma<1$, creating a payoff which is neither convex nor concave.

In the different models all possible scenarios with respect to the optimality of static or dynamic superhedges indeed occur. In the SI model with unbounded interest rates, only the static superhedge is optimal, whereas in the SJ model and in the SI model with non-negative interest rates all superhedges are dynamic. Finally in the SV economy there are both static and dynamic superhedges.

In the SV model the dominating payoff is shown in the left graph in Figure 1. It is composed of two 'pieces'. The first is given by the straight line which goes through the origin and which is tangent to the payoff graph. To the right of the tangency point the second piece is given by the claim itself. Since this claim is concave the associated superhedge in the worst-case model with zero volatility is dynamic. Corollary 1 shows that there is also a static superhedge. In the worst-case model there is no more uncertainty, so the strategy has to generate a payoff of $h\left(S_{0} e^{r T}\right)$ for the deterministic future stock price $S_{0} e^{r T}$, and the strategy has to dominate the payoff $h$ in the original model, which implies that the graph of the payoff of the strategy is tangent to the graph of the dominating concave payoff.

The dominating payoff in the SJ model is the same as in the case of SV. The superhedge is therefore also dynamic, and we can withdraw money whenever there is 
a jump. As we can see from Corollary 3 there is no static superhedge, since $\widehat{h}$ is not affine-linear.

Similar to the case described above the dominating payoff in the SI economy with unbounded interest rates is a straight line through the origin tangent to the payoff graph which is shown in the right graph in Figure 1. The reason why this tangent has to go through the origin is given in Proposition 3 which shows that the position will not contain an investment in the money market account. The superhedge in this case will be static, and Corollary 4 states that there will be no dynamic superhedging strategy.

If the interest rate is non-negative the dominating payoff is the same as in the case of SV. The replicating strategy in the worst-case model with a zero interest rate only precludes a short position in the money market account according to Proposition 4, but the claim would not require such a short position anyway. The superhedge is dynamic and for $R>0$, money can be withdrawn, which can be reinvested into an arbitrary limited liability strategy. Corollary 5 shows that there is no static superhedge.

\section{Bigger Models}

Bakshi, Cao, and Chen (1997) have proposed a model which simultaneously includes SV, SJ, and SI. Given that a user would like to apply such a model, the natural question is how superhedges for contingent claims should be set up when more than one non-traded risk factor is present. The superhedge will again be characterized by some optimal dominating claim, which has to meet all the restrictions derived for the respective risk factors. It will then be determined in a worst-case model which will also be derived from the worst-case models for the respective risk factors.

For example, in a model which includes both a jump component and an extra diffusion for volatility the dominating claim has to be concave, since it has to be concave in both individual models. The worst-case model will then be one without jumps and with a zero volatility. The stock price in this model is no longer stochastic, and as in the case 
of SV, there is always a static superhedge. Futhermore, if the dominating payoff is not affine-linear, there are also infinitely many dynamic superhedes.

When all three risk factors are present we have to distinguish between the cases of unbounded and non-negative interest rates. In the first scenario the dominating claim has to be concave in the SV and in the SJ economy and linear in the SI case. So in the combined model the dominating claim has to be linear. The worst case model will be one with no jumps and zero volatility. However, the hedging strategies will not contain investments in the money market account due to the reasons described in Subsection 2.4. Concerning the actual superhedges for the claims discussed in Section 3 we find that for the call the dominating claim is the stock with a static strategy. For the put there is no superhedge, which is caused by the impossibility to find such a hedge in the SI model. For the corporate bond the dominating claim is the stock with a static hedge, and for the power call with $\gamma<1$ the dominating claim is a linear tangent, also with a static hedge.

When interest rates are non-negative in this big model the dominating payoff has to be concave based on the SV and SJ model, and from the SI part we get the further restriction that the hedge cannot contain a short position in the money market account. The worst-case model is one with zero volatility, no jumps, and with a zero interest rate. For the call the dominating claim will again be the stock, whereas for the put it will be the constant payoff equal to the strike price, with the strategies being static in both cases. For the corporate bond the dominating claim will be the corporate bond itself with a dynamic hedge. Again, since the future stock price is certain in the worst-case model, there is also a static superhedge for the corporate bond. And for the power call with exponent $\gamma<1$, the dominating claim is equal to the dominating claim in the SV model.

\section{Summary and Conclusion}

The main contribution of this paper is the structural analysis of superhedges on various types of incomplete markets. The incompleteness can be caused by SV, SJ, SI, or an arbitrary combination of these three risk factors. Our particular interest has been in the 
question whether static or dynamic superhedging strategies are optimal for the contingent claims under consideration.

A key result of our investigation is that the optimality of the respective type of superhedge depends on both the model and the payoff of the claim. We provide an economic interpretation for the structure of optimal superhedges by showing that they can be characterized by dominating payoffs which have to meet certain model-dependent conditions for the implied strategy to be a candidate for a superhedge.

In the SV and SJ case the volatility of the underlying asset is the main item of interest. The worst case model eliminates (parts of) this volatility, since there is no more volatility risk in the SV economy, and no more jump risk in the SJ case. For the superhedges we only have to consider concave payoffs the prices of which decrease in volatility, and which are consequently maximal in the worst case model. In the SI model the behaviour of the short rate determines whether the hedge position can contain a long or a short position in the money market.

By construction, the worst case model is always one with a complete market. This is especially true for the SV economy, since here the worst-case model is even degenerate with a deterministic evolution of the stock price. In this special case we find that for certain types of claims both static and dynamic superhedges exist, which is not true for the other models.

As a common feature of all models we find that the existence of one dynamic superhedge implies that there are infinitely many such dynamic superhedges. The intuition here is that the strategy allows the withdrawal of funds from the superhedge portfolio in certain states of the world. These funds can then be invested in any limited liability strategy, and the new strategy will of course still be a superhedge. 


\section{References}

Avellaneda, M., A. Levy, and A. Parás, 1995, Pricing and hedging derivative securities in markets with uncertain volatilities, Applied Mathematical Finance 2, 73-88.

Bakshi, G., C. Cao, and Z. Chen, 1997, Empirical Performance of Alternative Option Pricing Models, Journal of Finance 52, 2003-2049.

Bergman, Y. Z., B. D. Grundy, and Z. Wiener, 1996, General Properties of Option Prices, Journal of Finance 51, 1573-1610.

Branger, N., A. Esser, and C. Schlag, 2003, Attainability of European Path-Independent Claims in Incomplete Markets, Working Paper, Goethe-Universität Frankfurt.

Cox, J. C., J. E. Ingersoll, and S. A. Ross, 1985, A theory of the term structure of interest rates, Econometrica 53, 385-407.

Cvitanic, J., H. Pham, and N. Touzi, 1999, Super-replication in stochastic volatility models with portfolio constraints, Journal of Applied Probability 36, 523-545.

Duffie, D., 2001, Dynamic Asset Pricing Theory. (3. Aufl., Princeton University Press Princeton).

Eberlein, E., and J. Jacod, 1997, On the range of option prices, Finance and Stochastics $1,131-140$.

Frey, R., and C. A. Sin, 1999, Bounds on European Option Prices under Stochastic Volatility, Mathematical Finance 9, 97-116.

Merton, R. C., 1973, Theory of Rational Option Pricing, Bell Journal of Economics and Management Science 4, 141-183.

Romano, M., and N. Touzi, 1997, Contingent Claims and Market Completeness in a Stochastic Volatility Model, Mathematical Finance 7, 399-412. 
Vasicek, O., 1977, An Equilibrium Characterization of the Term Structure, Journal of Financial Economics 5, 177-188. 


\begin{tabular}{|l|c|c|c|c|}
\hline & $\begin{array}{c}\text { Call } \\
\left(S_{T}-X\right)^{+}\end{array}$ & $\begin{array}{c}\text { Put } \\
\left(X-S_{T}\right)^{+}\end{array}$ & $\begin{array}{c}\text { Corporate Bond } \\
\min \left\{S_{T}, X\right\}\end{array}$ & $\begin{array}{c}\text { Power Call } \\
\left(S_{T}^{\gamma}-X\right)^{+}\end{array}$ \\
\hline SV & static & static & static + dynamic & static + dynamic \\
SJ & static & static & dynamic & dynamic \\
SIUSR & static & - & static & static \\
SINSR & static & dynamic & dynamic & dynamic \\
\hline
\end{tabular}

Table 1: Example: Structure of Superhedges

The table shows for four different claims and for several models whether the superhedge is static or dynamic or whether both strategies can be applied. $S_{T}$ is the terminal stock price, $X$ denotes the strike price, and the exponent $\gamma$ for the power call is less than one. 

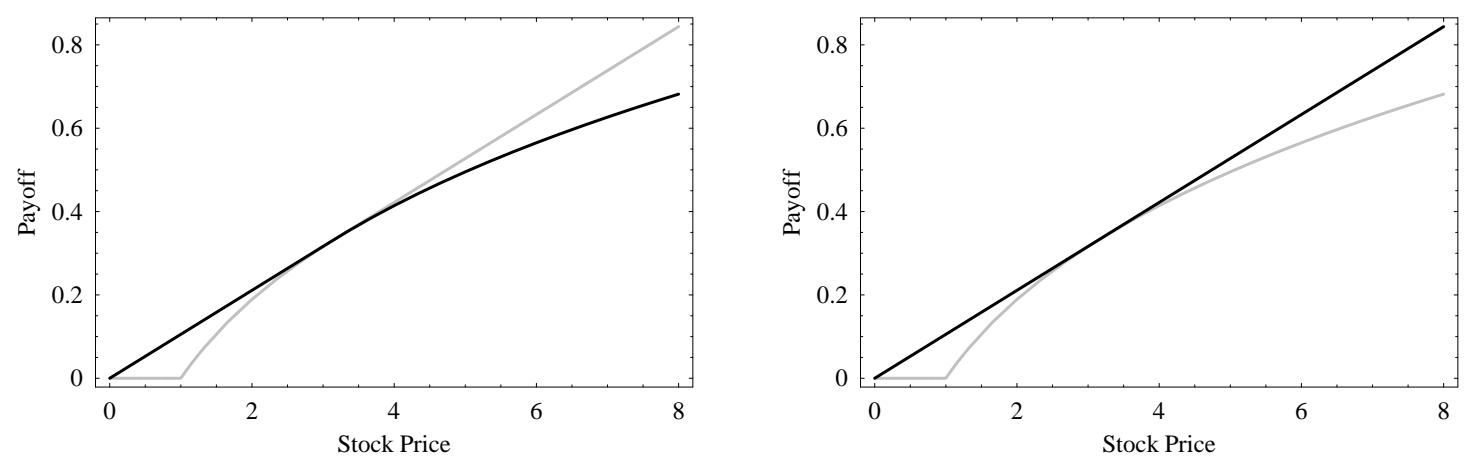

Figure 1: Power Call: Optimal dominating payoff $\widehat{h}$

Both graphs show the payoff of a power call with strike $X=1$ and exponent $\gamma=0.25$ as a function of the terminal stock price. The optimal dominating payoff for an SI model with non-negative interest rates, for an SJ model and for an SV model is given by the solid line in the left graph. The optimal dominating payoff for an SI model with unbounded interest rates is given by the solid line in the right graph. 